\title{
Modelling urban growth evolution and land-use changes using GIS based cellular automata and SLEUTH models: the case of Sana'a metropolitan city, Yemen.
}

\begin{abstract}
An effective and efficient planning of an urban growth and land use changes and its impact on the environment requires information about growth trends and patterns amongst other important information. Over the years, many urban growth models have been developed and used in the developed countries for forecasting growth patterns. In the developing countries however, there exist a very few studies showing the application of these models and their performances. In this study two models such as cellular automata (CA) and the SLEUTH models are applied in a geographical information system (GIS) to simulate and predict the urban growth and land use change for the City of Sana'a (Yemen) for the period 2004-2020. GIS based maps were generated for the urban growth pattern of the city which was further analyzed using geo-statistical techniques. During the models calibration process, a total of 35 years of time series dataset such as historical topographical maps, aerial photographs and satellite imageries was used to identify the parameters that influenced the urban growth. The validation result showed an overall accuracy of $99.6 \%$; with the producer's accuracy of $83.3 \%$ and the user's accuracy $83.6 \%$. The SLEUTH model used the best fit growth rule parameters during the calibration to forecasting future urban growth pattern and generated various probability maps in which the individual grid cells are urbanized assuming unique "urban growth signatures". The models generated future urban growth pattern and land use changes from the period 2004-2020. Both models proved effective in forecasting growth pattern that will be useful in planning and decision making. In comparison, the CA model growth pattern showed high density development, in which growth edges were filled and clusters were merged together to form a compact built-up area wherein less agricultural lands were included. On the contrary, the SLEUTH model growth pattern showed more urban sprawl and low-density development that included substantial areas of agricultural lands.
\end{abstract}

Keyword: Urban growth; Land-use change; Remote sensing; GIS; Cellular automata; SLEUTH; Sana`a 